BMJ Surgery, Interventions, $\&$ Health Technologies

\section{IDEAL Stage 2a experience with in- office, transperineal MRI/ultrasound software fusion targeted prostate biopsy}

To cite: Tzeng M, CriccoLizza E, Al Hussein Al Awamlh B, et al. IDEAL Stage 2a experience with in-office, transperineal MRI/ultrasound software fusion targeted prostate biopsy. BMJ Surg Interv Health Technologies 2019;1:e000025. doi:10.1136/ bmjsit-2019-000025

Received 09 September 2019 Revised 11 0ctober 2019 Accepted 25 0ctober 2019

Check for updates

(c) Author(s) (or their employer(s)) 2019. Re-use permitted under CC BY-NC. No commercial re-use. See rights and permissions. Published by BMJ.

${ }^{1}$ Department of Urology, Weill Cornell Medical College, New York City, New York, USA ${ }^{2}$ Department of Radiology, Weill Cornell Medical College, New York City, New York, USA

Correspondence to Dr Jim Hu; Jch9011@med.cornell.edu

\section{ABSTRACT}

Objective Although the feasibility of transperineal biopsy under local anesthesia has been demonstrated, little is known regarding the application of MRI/ultrasound software fusion targeted biopsy for transperineal biopsy under local anesthesia. The objective of our study is to describe our initial experience with in-office transperineal MRI/ultrasound software fusion targeted biopsy (Idea, Development, Exploration, Assessment, Long-term Followup [IDEAL] Stage 2a).

Methods Between October 2017 and July 2019, 33 men underwent in-office transperineal MRI-targeted biopsy using the Artemis (Eigen, Grass Valley, CA, USA) fixedrobotic arm system. The indication for biopsy was elevated prostate specific antigen (PSA) $(n=14)$, prior negative biopsy $(n=10)$, active surveillance $(n=6)$, and surveillance after partial gland cryoablation $(n=3)$. We prospectively captured patient demographic and clinical characteristics, biopsy outcomes, and complications. Complications were classified according to Common Terminology Criteria for Adverse Events (CTCAE) V.5.0.

Results The median patient age was 67 years (IQR 61-71) and the median serum PSA level was $7.0 \mathrm{ng} / \mathrm{mL}$ (IQR 5.1-11.4). The median duration of in-office MRItargeted transperineal biopsy was 26 min (IQR 23-28). Overall, transperineal MRI-targeted biopsy detected prostate cancer in $18(54.6 \%)$ men, with $8(24.2 \%)$ being clinically significant (Gleason Score $\geq 3+4$, Grade Group $\geq 2$ ). Clinically significant prostate cancer was detected in four $(28.6 \%)$ biopsy naïve men, two $(20.0 \%)$ men with a prior negative, one (16.7\%) man on active surveillance and one $(33.3 \%)$ man following partial gland ablation. Three (9.1\%) men experienced complications: two hematuria and one urinary retention.

Conclusion Our findings demonstrate the feasibility of the fixed-robotic arm fusion platform for in-office transperineal MRI-targeted biopsy and a low rate of adverse events. However, larger prostate size precludes MRI/ultrasound software fusion and pubic arch interference hindered the transperineal MRI-targeted approach in $9.1 \%$ of men. Pubic arch interference was overcome by a free-hand approach with software fusion guidance.

\section{INTRODUCTION}

The transrectal approach to prostate biopsy is the gold standard approach for detecting prostate cancer. However, a drawback of the

\section{Key messages}

What is already known about this subject?

- Transperineal biopsy, traditionally performed under general anesthesia, does not require antibiotic prophylaxis and has a significantly lower risk of infection compared with the traditional transrectal approach. There have been a few single-centre series that describe a transperineal approach under local anesthesia using ultrasound targeting (IDEAL 2) and one published series of transperineal MRI/ultrasound software fusion targeted biopsy performed under general anesthesia.

\section{What are the new findings?}

- This is the first series of in-office transperineal MRI/ ultrasound software fusion targeted prostate biopsy using local anesthesia. We demonstrate that cancer detection and complication rates are similar to transrectal MRI/ultrasound software fusion targeted biopsy outcomes with an infectious complication rate of $0 \%$ (without antibiotic prophylaxis).

\section{How might these results affect future}

\section{research or surgical practice?}

- We demonstrate that in-office MRI/ultrasound software fusion targeted biopsy is feasible and incorporates the advances of contemporary image guidance for greater accuracy as well as local anesthesia for widespread, cost-effective dissemination. Moreover, the significantly lower infection risk without antibiotic prophylaxis compared with the traditional transrectal approach improves patient outcomes as well as antibiotic stewardship.

transrectal approach is the translocation of rectal flora with each biopsy needle pass into the prostate, which contributes to infectious complications. The complication rate of urinary tract infections and sepsis has been reported to be as high as $5.2 \%$ and $3.1 \%$, respectively. ${ }^{12}$ With the recent rise in infectious complications to be as high as $7.0 \%$ of all transrectal biopsy, strategies to prevent infection are of paramount importance. ${ }^{2}$

The transperineal approach is a percutaneous technique for prostate biopsy needle 
passage that avoids translocating rectal bacteria into the sterile urinary tract altogether. The rate of cancer detection with transperineal biopsy is similar to transrectal biopsy. ${ }^{3-6}$ Systematic reviews of transperineal biopsy under general and local anesthesia also demonstrate lower rates of infectious complication, with reported rates of urinary tract infection and sepsis as low as $0 \%-1.6 \%$ and $0 \% .{ }^{78}$ However, with transperineal prostate biopsy under general anesthesia, many men received intravenous antibiotic prophylaxis. Moreover, some studies demonstrate similar rates of infectious and non-infectious complications between transperineal and transrectal biopsies. ${ }^{5}$ Whereas transrectal biopsies are commonly performed with local anesthesia, transperineal biopsies have traditionally been performed under general anesthesia to avoid patient discomfort. ${ }^{5} 7$ This practice brings into question the clinical utility of transperineal biopsy as a ubiquitous approach, as general anesthesia is more costly and has associated risks. Moreover, transperineal biopsy under general anesthesia is typically reserved for saturation biopsy after prior negative biopsies.

The use of transperineal biopsy with ultrasound guidance under local anesthesia has been described in a few studies, attaining IDEAL Stage $2 b .{ }^{9-11}$ However, there is only one series that assesses the efficacy of a commercially available transperineal MRI/ultrasound software fusion targeted biopsy platform. In this IDEAL Stage 2a series, 32 men underwent general anesthesia for transperineal MRI/ultrasound fusion targeted biopsy. ${ }^{12}$ Another study exists that evaluates transperineal MRI/ultrasound targeted biopsy using cognitive rather than software fusion. ${ }^{13}$

There is recent high-level evidence demonstrating the superiority of MRI/ultrasound fusion targeted biopsy over ultrasound-guided biopsy. ${ }^{14}$ Moreover, there is greater detection of clinically significant prostate cancer with MRI/ultrasound software fusion over cognitive fusion during transrectal biopsy. ${ }^{15}$ Given these benefits with MRI/ultrasound software fusion targeting during transrectal biopsy, our objective is to describe our technique and assess early outcomes for transperineal MRI/ ultrasound software fusion targeted (henceforth MRItargeted) prostate biopsy under local anesthesia (IDEAL Stage 2a). Such studies are needed to evaluate the potential for widespread adoption of in-office, transperineal MRI-targeted biopsy.

\section{METHODS}

Data were prospectively collected for 33 men who underwent in-office transperineal MRI-targeted biopsy during October 2017 to July 2019 with the Artemis platform (Eigen, Grass Valley, CA, USA). Our study was approved by the Weill Cornell Institutional Review Board. Patients were informed as part of the consent process that the transperineal approach is associated with lower risk of infections; however, the novel use of an MRI-targeted approach had yet to be studied with this biopsy approach.

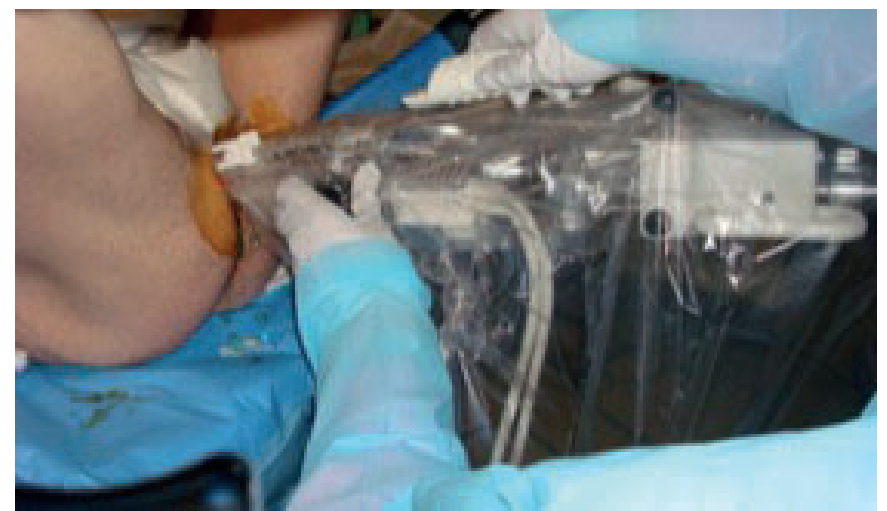

Figure 1 In-office transperineal probe placement utilizing the Artemis MRI-ultrasound fusion platform for prostate biopsy under local anesthesia.

Moreover, patients were informed that evidence demonstrates a similar rate of cancer detection using the transperineal approach under local anesthesia. ${ }^{9} 16$

Men underwent a Fleet enema to optimize transrectal ultrasound visualization of the prostate; however, no prophylactic antibiotics were given. Patients were positioned into the lithotomy position with yellow-fin stirrups supporting the knee and hips at right angles. A surgical towel was used to tape the scrotum anteriorly, and the perineum was shaved and prepped with chlorhexidine (figure 1). For the local anesthetic block, $10 \mathrm{~mL}$ of $1 \%$ lidocaine was injected into the perineal subcutaneous tissue. In all, $60 \mathrm{~mL}$ of lube was then injected into the rectum prior to insertion of the ultrasound probe to guide the deeper anesthetic block. The Hitachi C41L47RP endorectal transducer (Hitachi Medical Systems America, Twinsburg, OH, USA) was then latched to the Artemis cradle and paired with the Hitachi Noblus ultrasound system. We then performed the anesthetic block of the pelvic floor and prostate apex as described by Kubo et al. ${ }^{17}$ The Artemis ultrasound cradle was then rotated away from the midline bilaterally to visualize the pelvic floor muscles and the interface between the apex of the prostate and the pelvic floor where a total of $10 \mathrm{~mL}$ of $1 \%$ lidocaine was injected. A total of $20 \mathrm{~mL}$ of $1 \%$ lidocaine was used.

The ultrasound probe was rotated using the Artemis cradle to scan the prostate. The Artemis assembled the ultrasound images and segmentation of the prostate was performed. DJM performed prostate segmentation prior to biopsy and the MRI was incorporated into the TRUS through image registration, similar to transrectal MRItargeted biopsy. ${ }^{18}$ The Artemis transperineal system has 2 degrees of freedom of movement. These include rotation of the probe within the Artemis cradle, and needle angulation along a fulcrum mounted on top of the carriage to hold the ultrasound probe in place. Prior to biopsy, the carriage is advanced such that the needle guide is in contact with the perineal skin. Using a combination of these two movements, targeted biopsies of regions of interest (ROI) were performed first (figure 2), followed by systematic biopsies. 


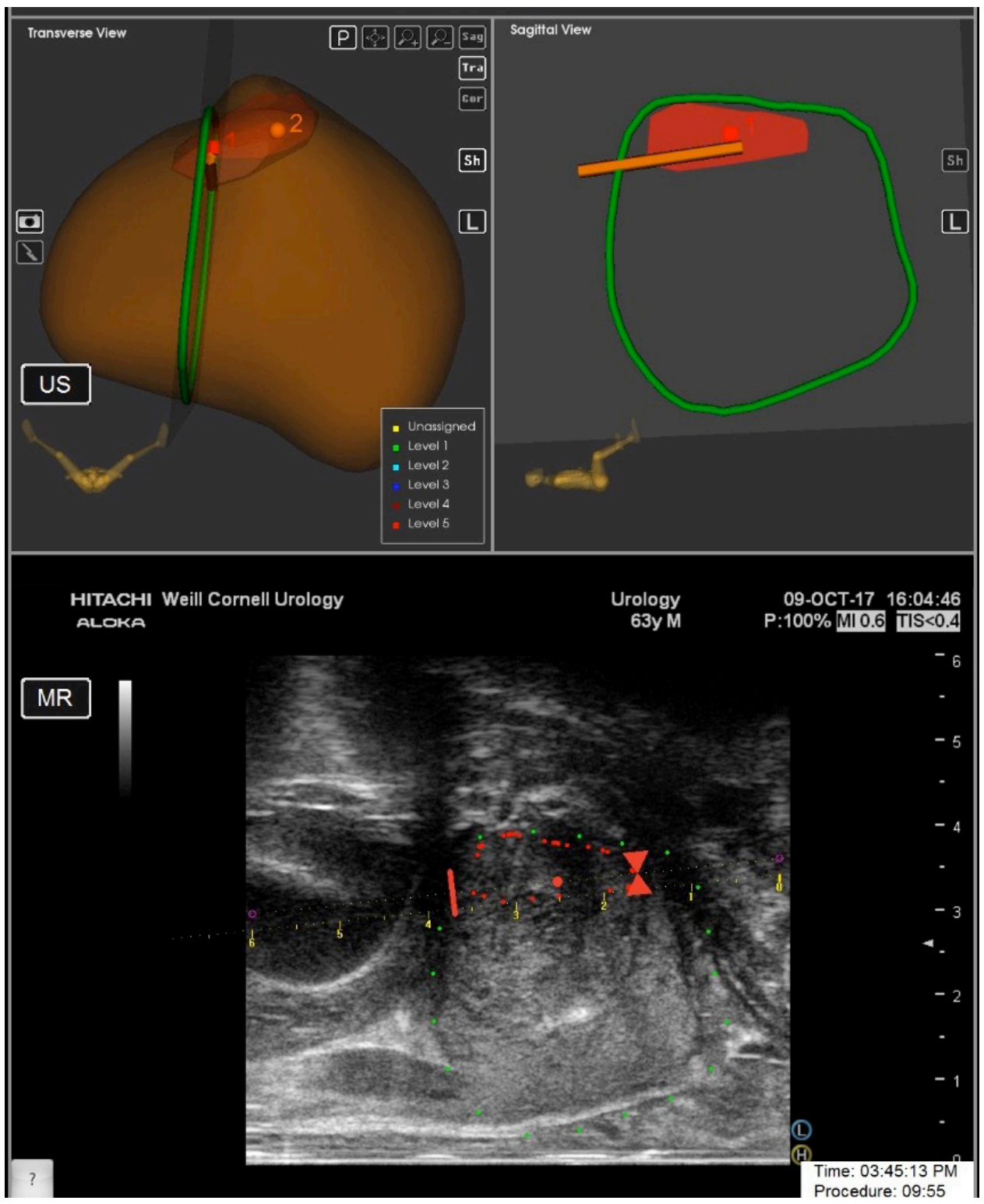

Figure 2 Transperineal MRI/ultrasound software fusion and targeting of an anterior Prostate Imaging-Reporting and Data System four lesion for biopsy.

Prospectively captured data included patient demographics, duration of transperineal biopsy, prostate specific antigen (PSA), MRI findings, biopsy results, and complications. PSA values for three men were unavailable. We measured pain using a visual analogue scale (VAS) in the first 10 subjects. ${ }^{19}$ After ensuring tolerability, this was not continued. Complications were classified according to Common Terminology Criteria for Adverse Events (CTCAE) V.5.0. ${ }^{20}$

\section{Technical modifications}

Initially, we performed the prostate anesthetic block along the neurovascular bundles, injecting from base to apex. However, for Case 8, we adopted the technique described by Kubo et al and continued with this anesthetic approach. ${ }^{17}$ Additionally, we initially used an endocavity balloon (Civco Coralville, Iowa, USA) to cover the ultrasound transducer. However, at Case 16, we switched to applying $60 \mathrm{~mL}$ of lube using a catheter tip syringe, which achieved a similar effect at greater efficiency and lower cost.

The Artemis software froze following acquisition of targeted biopsy cores for one man (Case 15), requiring the systematic cores to be performed free-hand. In three men (Cases 16, 18, and 32), pubic arch interference prevented the acquisition of some of the systematic cores while it prevented targeted biopsy in another, who was excluded from analysis (table 1). In these three cases, the ultrasound carriage was unclasped to flip the needle guide away and biopsies were performed freehand with the ultrasound probe resting in the Artemis cradle. In one patient with a $253 \mathrm{~mL}$ prostate, the prostate was too large to be captured completely in one sagittal view of the ultrasound, and therefore MRI-ultrasound software fusion could not be performed. In this patient, cognitive MRI targeting and free-hand systematic biopsy 


\begin{tabular}{|c|c|c|c|}
\hline Case & Duration (min) & Complications & Technical problems \\
\hline 1 & 32 & & \\
\hline 2 & 29 & & \\
\hline 3 & 31 & & $\begin{array}{l}\text { Significant needle } \\
\text { deflection }\end{array}$ \\
\hline 4 & 29 & & \\
\hline 5 & 28 & & \\
\hline 6 & 30 & Hematuria & \\
\hline 7 & 27 & & \\
\hline 8 & 28 & & \\
\hline 9 & 27 & & \\
\hline 10 & 29 & & \\
\hline 11 & 26 & & \\
\hline 12 & 27 & Hematuria & \\
\hline 13 & 25 & & Artemis software froze \\
\hline 14 & 28 & & \\
\hline 15 & 24 & & \\
\hline 16 & 26 & & Pubic arch interference \\
\hline 17 & 25 & & \\
\hline 18 & 28 & & Pubic arch interference \\
\hline 19 & 24 & & \\
\hline 20 & 26 & & \\
\hline 21 & 28 & & \\
\hline 22 & 23 & & \\
\hline 23 & 25 & & \\
\hline 24 & 23 & & \\
\hline 25 & 22 & $\begin{array}{l}\text { Urinary retention } \\
\text { requiring } \\
\text { catheterization }\end{array}$ & \\
\hline 26 & 23 & & \\
\hline 27 & 25 & & \\
\hline 28 & 20 & & \\
\hline 29 & 22 & & \\
\hline 30 & 21 & & \\
\hline 31 & 24 & & Pubic arch interference \\
\hline 32 & 20 & & \\
\hline 33 & 21 & & \\
\hline
\end{tabular}

were performed. This patient was also excluded from analysis. Finally, care must be taken regarding the bevel orientation of the biopsy needle relative to the sagittal ultrasound-imaging plane. The bevel tip cuts tissue at an angle and deflects in the direction of the bevel. This may be overcome with the use of symmetric tip biopsy needles.

\section{RESULTS}

The median age was 67 years (IQR 61-71), median PSA was $7.0 \mathrm{ng} / \mathrm{mL}$ (IQR 5.1-11.4), and median prostate volume was $47 \mathrm{~mL}$ (IQR 39-66). Indication for prostate biopsy was an elevated PSA in 14, prior negative biopsy in 10, active surveillance in 6 and surveillance following partial gland ablation in 3 . The median number of systematic and targeted cores sampled per patient were 9 (IQR 2-12) and 4 (IQR 2-6), respectively. A total of 47 ROI in 32 patients were demonstrated on MRI using the Prostate Imaging-Reporting and Data System (PI-RADS) v2.0: 16 (34.0\%) were PI-RADS 3, 25 (53.2\%) were PI-RADS 4, and $6(12.8 \%)$ were PI-RADS 5 . One post-partial gland cryoablation patient did not have ROI on post-ablation MRI. In this case, the treated areas underwent targeted biopsy using the pretreatment MRI along systematic biopsy (table 2). The two other post-partial gland cryoablation patients obtained biopsies in the context of targeting suspicious MRI findings (PI-RADS 4 and 5).

The majority $(\mathrm{n}=27,81.8 \%)$ of men underwent both systematic and targeted biopsies; however, six patients underwent targeted biopsy alone: two had a prior negative, two were on active surveillance for low-grade cancer diagnosed 12 months prior, and two were biopsy naive (table 3). Only targeted cores were taken from the biopsy naïve patients due to older age (1) and patient preference (1).

The median duration of in-office MRI-targeted transperineal biopsy was $26 \mathrm{~min}$ (IQR 23-28). A subanalysis of the 27 men who underwent both targeted and systematic biopsy also showed a median duration of $26 \mathrm{~min}$ (IQR 23-28). The median VAS pain score during the first 10 cases in the series was 3 (IQR 2-4).

Overall, transperineal MRI-targeted biopsy detected prostate cancer in $18(54.6 \%)$ men, with 8 (24.2\%) being clinically significant (Gleason Score $\geq 3+4$, Grade Group $\geq 2$ ). Clinically significant prostate cancer was detected in four $(28.6 \%)$ biopsy naive men, two $(20.0 \%)$ men with a prior negative biopsy, one (16.7\%) man on active surveillance and one $(33.3 \%$ ) man following partial gland ablation. Indolent prostate cancer was detected in five $(35.7 \%)$ biopsy naive men, three $(30.0 \%)$ men with a prior negative biopsy, one (16.7\%) man on active surveillance and one $(33.3 \%)$ man following partial gland ablation.

In biopsy naïve men, a Gleason Grade Group one was detected in five $(35.7 \%)$ patients. The detection rates for clinically significant prostate cancer for PI-RADS 3, 4, and 5 lesions in biopsy naïve men were $0 \%, 42.9 \%$, and $33.3 \%$, respectively.

Overall, three $(9.1 \%)$ patients experienced complications: two $(6.1 \%)$ patients reported hematuria (CTCAE Grade 1). Of note, this was hematuria in which patients contacted the provider to report; patients were reassured without sequelae. One $(3.0 \%)$ patient experienced urinary retention requiring catheterization in the emergency room (CTCAE Grade 2). There were no infectious complications (table 1).

\section{DISCUSSION}

To the best of our knowledge, this is the first series of in-office transperineal MRI-targeted prostate biopsy using local anesthesia. Overall and clinically significant prostate 
Table 2 Patient demographics, MRI results, and biopsy results

\begin{tabular}{|c|c|}
\hline Total patients & 33 \\
\hline Patients with any prostate cancer, $\mathrm{n}(\%)$ & $18(54.6)$ \\
\hline $\begin{array}{l}\text { Patients with clinically significant prostate } \\
\text { cancer, } n(\%)\end{array}$ & $8(24.2)$ \\
\hline Median (IQR) prebiopsy PSA level, ng/mL & $7.0(5.1-11.4)$ \\
\hline Median (IQR) prebiopsy prostate volume, $\mathrm{mL}$ & $47(39-66)$ \\
\hline Median (IQR) age, years & $67(59-71)$ \\
\hline \multicolumn{2}{|l|}{ Race/ethnicity, n (\%) } \\
\hline Decline & $11(33.3)$ \\
\hline White & $11(33.3)$ \\
\hline Black & $2(6.1)$ \\
\hline Asian & $3(9.9)$ \\
\hline Other & $6(18.2)$ \\
\hline \multicolumn{2}{|l|}{ Clinical history, $\mathrm{n}(\%)$} \\
\hline Biopsy naïve & $14(42.4)$ \\
\hline Active surveillance & $6(18.2)$ \\
\hline Prior negative biopsy & $10(30.3)$ \\
\hline Post-partial gland cryoablation & $3(9.1)$ \\
\hline Median (IQR) systematic cores taken & $9(2-12)$ \\
\hline Median (IQR) targeted cores taken & $4(2-6)$ \\
\hline \multicolumn{2}{|l|}{ Tumor characteristics } \\
\hline Number of ROls, total & 47 \\
\hline \multicolumn{2}{|l|}{ MRI Grades (PI-RADS v2 Score), total, n (\%) } \\
\hline 3 & $16(34.0)$ \\
\hline 4 & $25(53.2)$ \\
\hline 5 & $6(12.8)$ \\
\hline \multicolumn{2}{|l|}{ Highest Gleason Grade Group, n (\%) } \\
\hline Benign & $15(45.6)$ \\
\hline 1 & $10(30.3)$ \\
\hline 2 & $6(18.2)$ \\
\hline 3 & $1(3.0)$ \\
\hline 4 & $0(0)$ \\
\hline 5 & $1(3.0)$ \\
\hline Complications, n (\%) & $3(9.1)$ \\
\hline Hematuria & $2(6.1)$ \\
\hline Urinary retention & $1(3.0)$ \\
\hline
\end{tabular}

PI-RADS, Prostate Imaging-Reporting and Data System; PSA, prostate specific antigen.

cancer detection rates of $54.6 \%$ and $24.2 \%$ were observed. These findings are similar to the previously published detection rates of transrectal MRI-targeted biopsies and support evidence demonstrating that transperineal biopsies have fewer infectious complications. ${ }^{15}$

Sources of inaccuracy during MRI-targeted biopsy include image registration as well as patient movement. During transrectal MRI-targeted biopsy with the Artemis platform, there is considerable movement and pressure of the end-fire ultrasound probe on the prostate that causes deformation of the prostate shape. This is
Table 3 Types of cores taken, by clinical history.

\begin{tabular}{lll}
\hline Clinical history & $\begin{array}{l}\text { Targeted+ } \\
\text { systematic }\end{array}$ & $\begin{array}{l}\text { Targeted } \\
\text { only }\end{array}$ \\
\hline Biopsy naïve & 12 & 2 \\
Active surveillance & 4 & 2 \\
Prior negative biopsy & 8 & 2 \\
Post-partial gland cryoablation & 3 & 0 \\
\hline
\end{tabular}

overcome by software elastic registration. In contrast, with the transperineal MRI-targeted biopsy approach, the only movement of the ultrasound probe is rotation. However, the distance to targets from the skin is farther than the distance from the rectum to targets during transperineal versus transrectal MRI-targeted biopsy. Patient motion during transperineal MRI-targeted biopsy is overcome by motion compensation, which allows real-time adjustment of MRI-ultrasound image registration. This is similar to transrectal MRI-targeted biopsy using the Artemis platform.

In regard to adverse events, there were no infectious complications and only one man $(3.0 \%)$ experienced urinary retention. This aligns with the $1.6 \%$ to $11.4 \%$ rate of urinary retention that has been previously described by the National Institute for Health and Clinical Excellence. $^{21}$ There were no complaints of perineal bruising or wound issues. Transperineal biopsies may be the preferred approach in patients with high risk of infection and should be considered in light of increasing rates of infectious complications after transrectal biopsies. ${ }^{22}{ }^{23}$ Some studies report the mean duration of transrectal MRI-targeted biopsies to be 20 min. $^{24} 25$ This offers an advantage compared with transperineal MRI-targeted biopsies, which we found to have a median duration of 26 min.

Despite refinements to transperineal biopsy, significant challenges must be surmounted before widespread adoption. MRI-targeted transperineal biopsy systems cost \$150000-\$250 000 and their outcomes remain unproven. Additionally, in vivo trials demonstrating efficacy were not required for Food and Drug Administration approval of transperineal MRI-targeted platforms, due to the existence of a predicate device. This is noteworthy, given the greater distance the transperineal biopsy needle must traverse through the skin, subcutaneous fat, Colles' fascia and the pelvic floor muscles to reach the prostate as compared with transrectal biopsy. The greater distance and traversing more anatomic structures pose significant challenges in terms of accurate targeting. For instance, an in vivo study demonstrated an average needle deflection error of $9 \mathrm{~mm}$ with the MRI-targeted transperineal approach. ${ }^{26}$ The median maximal diameter of targets on MRI-targeted biopsy is $1.2 \mathrm{~mm} .{ }^{27}$ In contrast, studies of transrectal targeted biopsy platforms demonstrate an error distance of $1-3 \mathrm{~mm}$ from the biopsy needle to the ROI. $^{18}$ 
Our findings must be interpreted in the context of the study design. This is an early, single-arm series that demonstrates feasibility of the fixed robotic tracking arm Artemis system for MRI-targeted biopsy. The indication for biopsy was varied and larger numbers are needed to compare to published transrectal MRI-targeted biopsy series. For instance, a significant proportion of men in the study had a prior negative biopsy, were on active surveillance or underwent prior partial gland cryoablation so the overall detection rate of clinically significant prostate cancer is lower that what may be expected in a pure series of biopsy naïve patients. Moreover, the challenges observed with pubic arch interference and the inability to scan and segment larger prostates may be overcome with future version of MRI-targeted transperineal biopsy platforms. This raises the question of what is the learning curve for this procedure. However, we previously reported a learning curve of 98 cases for transrectal MRItargeted biopsy. ${ }^{28}$ Moreover, JCH performed more than 750 MRI-targeted transrectal biopsies, and the software interface for MRI-ultrasound fusion with the transperineal approach is the same as transrectal. Therefore, there was no learning curve for interfacing with software image registration during transperineal MRI-targeted approach. Future IDEAL Stage 2b studies for transperineal MRItargeted biopsy will define the learning curve for this novel procedure and explore the impact of needle deflection on the accuracy of transperineal MRI-targeted biopsy. Finally, although we did not abort any procedures due to pain or discomfort, we did not measure patient-reported outcomes measures in all men. Kubo et al demonstrated similar pain for transrectal and transperineal biopsy under local anesthesia. ${ }^{17}$ Prospective, comparative studies during MRI-targeted biopsy are needed.

\section{CONCLUSION}

In-office transperineal MRI-targeted prostate biopsy using local anesthesia appears to be a feasible approach with few complications that builds on the in-office approach to transperineal biopsy that has been described. Comparative studies are needed with the transrectal-targeted biopsy approach, and quantitative studies regarding needle deflection and sampling error are needed to refine the approach. Finally, our findings have significant implications for MRI-targeted transperineal partial gland ablation approaches that employ laser, cryotherapy, and other energy sources. Similar challenges such as pubic arch interference and obstacles to MRI-ultrasound software fusion, such as large prostate size, must be overcome for accurate and successful ablation.

\section{Twitter Jim Hu @jimhumd}

Contributors All authors have contributed significantly to the study design, collection of data, analysis, and/or manuscript preparation.

Funding This work was supported by the Frederick J. and Theresa Dow Wallace Fund of the New York Community Trust.

Competing interests None declared.
Patient consent for publication Not required.

Provenance and peer review Not commissioned; externally peer reviewed.

Data availability statement No data are available.

Open access This is an open access article distributed in accordance with the Creative Commons Attribution Non Commercial (CC BY-NC 4.0) license, which permits others to distribute, remix, adapt, build upon this work non-commercially, and license their derivative works on different terms, provided the original work is properly cited, appropriate credit is given, any changes made indicated, and the use is non-commercial. See: http://creativecommons.org/licenses/by-nc/4.0/.

ORCID iD

Michael Tzeng http://orcid.org/0000-0003-1384-407X

\section{REFERENCES}

1 Simsir A, Kismali E, Mammadov R, et al. Is it possible to predict sepsis, the most serious complication in prostate biopsy? Urol Int 2010;84:395-9.

2 Liss MA, Ehdaie B, Loeb S, et al. An update of the American urological association white paper on the prevention and treatment of the more common complications related to prostate biopsy. J Urol 2017;198:329-34.

3 Kawakami S, Yamamoto S, Numao N, et al. Direct comparison between transrectal and transperineal extended prostate biopsy for the detection of cancer. Int J Urol 2007;14:719-24.

4 Lo KL, Chui KL, Leung CH, et al. Outcomes of transperineal and transrectal ultrasound-guided prostate biopsy. Hong Kong Med J 2019;25:209-15

5 Xue J, Qin Z, Cai H, et al. Comparison between transrectal and transperineal prostate biopsy for detection of prostate cancer: a meta-analysis and trial sequential analysis. Oncotarget 2017;8:23322-36.

6 Shen P-F, Zhu Y-C, Wei W-R, et al. The results of transperineal versus transrectal prostate biopsy: a systematic review and metaanalysis. Asian J Androl 2012;14:310-5.

7 Chang DTS, Challacombe B, Lawrentschuk N. Transperineal biopsy of the prostate-is this the future? Nat Rev Urol 2013;10:690-702.

8 Loeb S, Vellekoop A, Ahmed HU, et al. Systematic review of complications of prostate biopsy. Eur Urol 2013;64:876-92.

9 Meyer AR, Joice GA, Schwen ZR, et al. Initial experience performing In-office ultrasound-guided Transperineal prostate biopsy under loca anesthesia using the PrecisionPoint Transperineal access system. Urology 2018;115:8-13.

10 Stefanova V, Buckley R, Flax S, et al. Transperineal prostate biopsies using local anesthesia: experience with 1,287 patients. prostate cancer detection rate, complications and patient tolerability. J Urol 2019;201:1121-6.

11 McCulloch P, Altman DG, Campbell WB, et al. No surgical innovation without evaluation: the ideal recommendations. Lancet 2009;374:1105-12

12 Kosarek CD, Mahmoud AM, Eyzaguirre EJ, et al. Initial series of magnetic resonance imaging (MRI)-fusion targeted prostate biopsy using the first transperineal targeted platform available in the USA. BJU Int 2018;122:909-12.

13 Bass EJ, Donaldson IA, Freeman A, et al. Magnetic resonance imaging targeted transperineal prostate biopsy: a local anaesthetic approach. Prostate Cancer Prostatic Dis 2017;20:311-7.

14 Kasivisvanathan V, Rannikko AS, Borghi M, et al. MRI-Targeted or standard biopsy for prostate-cancer diagnosis. N Engl J Med 2018;378:1767-77.

15 Elkhoury FF, Felker ER, Kwan L, et al. Comparison of targeted vs systematic prostate biopsy in men who are biopsy naive: the prospective assessment of image registration in the diagnosis of prostate cancer (PAIREDCAP) study. JAMA Surg 2019. doi:10.1001/ jamasurg.2019.1734. [Epub ahead of print: 12 Jun 2019].

16 Ristau BT, Allaway M, Cendo D, et al. Free-hand transperineal prostate biopsy provides acceptable cancer detection and minimizes risk of infection: evolving experience with a 10-sector template. Urol Oncol 2018;36:528.e15-20.

17 Kubo Y, Kawakami S, Numao N, et al. Simple and effective local anesthesia for transperineal extended prostate biopsy: application to three-dimensional 26-core biopsy. Int J Urol 2009;16:420-3.

18 Natarajan S, Marks LS, Margolis DJA, et al. Clinical application of a 3D ultrasound-guided prostate biopsy system. Urol Oncol 2011;29:334-42.

19 Downie WW, Leatham PA, Rhind VM, et al. Studies with pain rating scales. Ann Rheum Dis 1978;37:378-81. 
20 Institute DoHaHSNIoHNC. Common terminology criteria for adverse events v5.0, NCl, NIH. DHHS 2017.

21 Excellence NloHaC. Interventional procedures programme: Interventional procedure overview of transperineal template biopsy and mapping of the prostate. [IP793] 2009.

22 Carignan A, Roussy J-F, Lapointe V, et al. Increasing risk of infectious complications after transrectal ultrasound-guided prostate biopsies: time to reassess antimicrobial prophylaxis? Eur Urol 2012;62:453-9.

23 Emiliozzi P, Longhi S, Scarpone P, et al. The value of a single biopsy with 12 transperineal cores for detecting prostate cancer in patients with elevated prostate specific antigen. J Urol 2001;166:845-50.

24 Sonn GA, Chang E, Natarajan S, et al. Value of targeted prostate biopsy using magnetic resonance-ultrasound fusion in men with prior negative biopsy and elevated prostate-specific antigen. Eur Urol 2014;65:809-15.
25 Sonn GA, Natarajan S, Margolis DJA, et al. Targeted biopsy in the detection of prostate cancer using an office based magnetic resonance ultrasound fusion device. J Urol 2013;189:86-92.

26 Cepek J, Chronik BA, Lindner U, et al. A system for MRI-guided transperineal delivery of needles to the prostate for focal therapy. Med Phys 2013;40:012304.

27 Patel N, Halpern JA, Kasabwala K, et al. Multiple regions of interest on multiparametric magnetic resonance imaging are not associated with increased detection of clinically significant prostate cancer on fusion biopsy. J Urol 2018;200:559-63.

28 Kasabwala K, Patel N, Cricco-Lizza E, et al. The learning curve for magnetic resonance Imaging/Ultrasound Fusion-guided prostate biopsy. Eur Urol Oncol 2019;2:135-40. 